\title{
Crioablación en Taquicardia ortodrómica por vía accesoria oculta parahisiana: caso clínico y revisión de la literatura.
}

\author{
Rodrigo Isa, Mauricio Moreno1, Jorge Palazzolo. \\ Hospital El Carmen de Maipú, Santiago de Chile \\ 1 Clínica Dávila, Santiago de Chile
}

\section{Crioablation of orthodromic supraventricular tachycardia win an occult accessory pathway}

An 18-year-old woman with recurrent tachycardia was shown to have orthodromic supraventricular tachycardia through an antero-septal occult pathway near the His. Cryoablation was selected to avoid A-V block. The accesory pathway was finally interrupted after $360 \mathrm{sec}$ of cryoablation. No recurrence was documented after a 12 month follow-up

Key words: Cryoablation; arrhythmia; tachycardia. 


\section{Caso clínico:}

Mujer de 18 años sin antecedentes mórbidos relevantes que presenta desde los 15 años episodios frecuentes de taquicardia paroxística supraventricular, algunas veces semanalmente, con múltiples consultas al servicio de urgencia. Algunos episodios son autolimitados y otros son interrumpidos con adenosina y verapamilo endovenoso. Fue derivada al servicio de electrofisiología del hospital El Carmen de Maipú.

Luego de explicar la patología a la paciente y su familia, y una vez obtenido el consentimiento informado, se realizó un estudio electrofisiológico convencional: punción venosa femoral derecha triple con introductores 7 fr. Catéter decapolar en seno coronario, catéter cuadripolar en His y otro en ventrículo derecho. Se obtuvo registro intracavitario en ritmo sinusal con intervalos basales normales (AH: $80 \mathrm{~ms}$ y HV: $40 \mathrm{~ms}$ ). En forma espontánea se indujo taquicardia regular de QRS

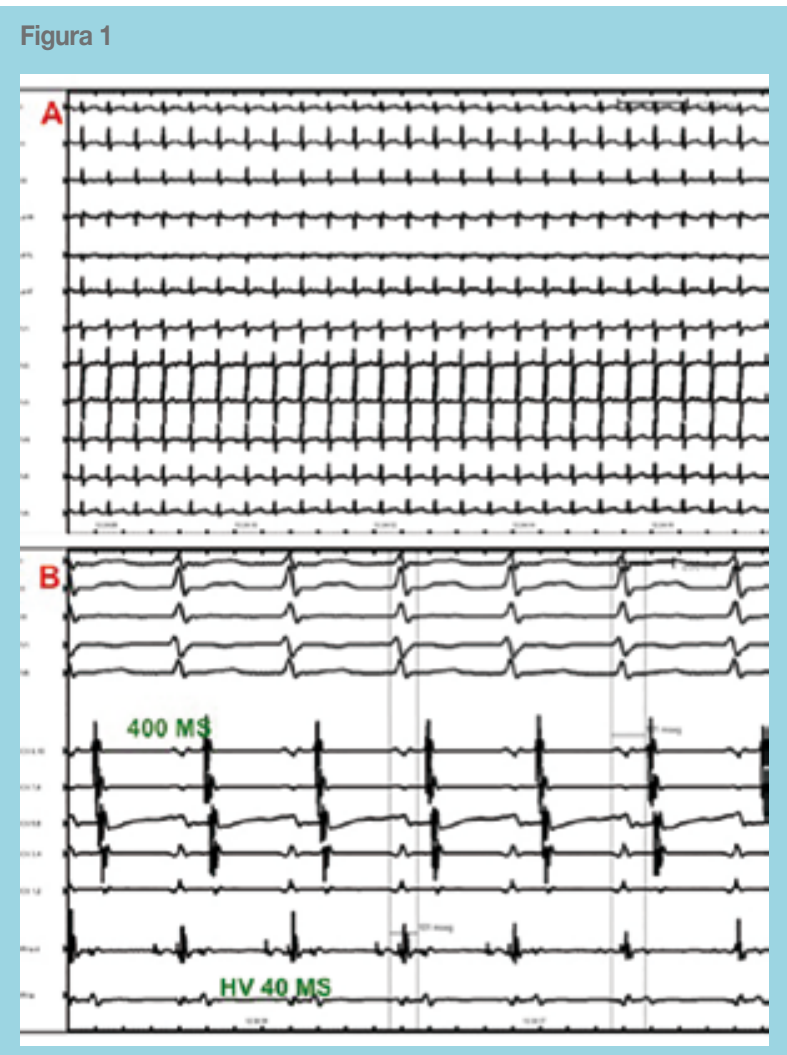

A. ECG de 12 derivaciones en taquicardia regular de QRS estrecho con onda P visible sobre la onda T.

B. Registro intracavitario durante la misma taquicardia. Los 5 canales superiores corresponden a derivaciones ECG I, II, III, VI y V6, del sexto al décimo canal, el registro de seno coronario desde proximal a distal y los dos canales inferiores, al registro de His. La taquicardia tiene un ciclo de $400 \mathrm{~ms}$, un intervalo HV de $40 \mathrm{~ms}$, un intervalo VA septal de $100 \mathrm{~ms}$ y un VA en seno coronario proximal de $120 \mathrm{~ms}$. estrecho y relación VA 1:1 y ciclo de 400 ms compatible con una taquicardia supraventricular. El intervalo VA durante taquicardia fue de $100 \mathrm{~ms}$ con electrograma auricular más precoz a nivel de His. El intervalo VA a nivel de ostium de SC fue $120 \mathrm{~ms}$ (Figura $1 \mathrm{~A} \mathrm{y} \mathrm{B}$ ).

En forma espontánea se observó interrupción de la taquicardia con onda P final. La introducción de un extraestímulo ventricular durante taquicardia, interrumpe ésta sin capturar aurícula. Ambas observaciones hacen muy improbable que el mecanismo de esta taquicardia supraventricular sea una taquicardia auricular (Figura 2A y B).

Se realizaron maniobras electrofisiológicas de diagnóstico diferencial de TPSV, durante taquicardia y ritmo sinusal que concluyeron en el diagnóstico de una taquicardia ortodrómica mediada por una vía accesoria oculta de localización anteroseptal (Figura 3A, B, C y D). La cartografía de activación del anillo tricuspídeo sep-

Figura 2

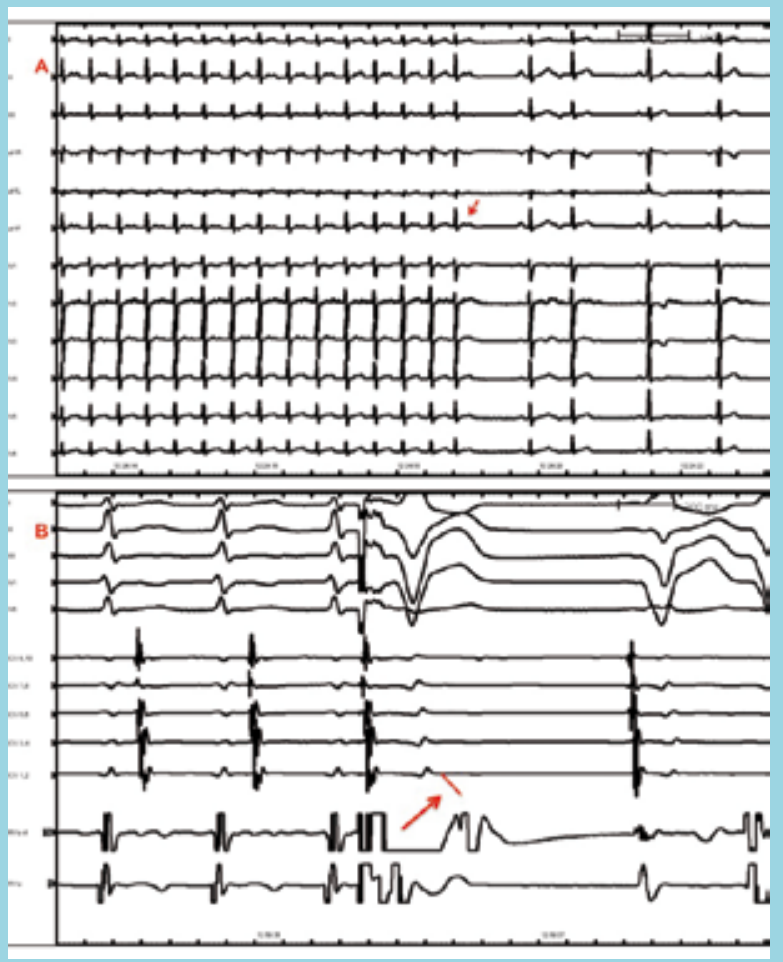

A. ECG de 12 derivaciones durante interrupción espontánea de la taquicardia. Flecha roja indica onda $P$.

B. Registro intracavitario durante taquicardia. Los 10 canales superiores siguen misma disposición que figura $2 B$ y los dos canales inferiores registran el ápex de ventrículo derecho. La flecha y línea roja indican la interrupción de la taquicardia tras un extraestímulo ventricular.. 
Figura 3

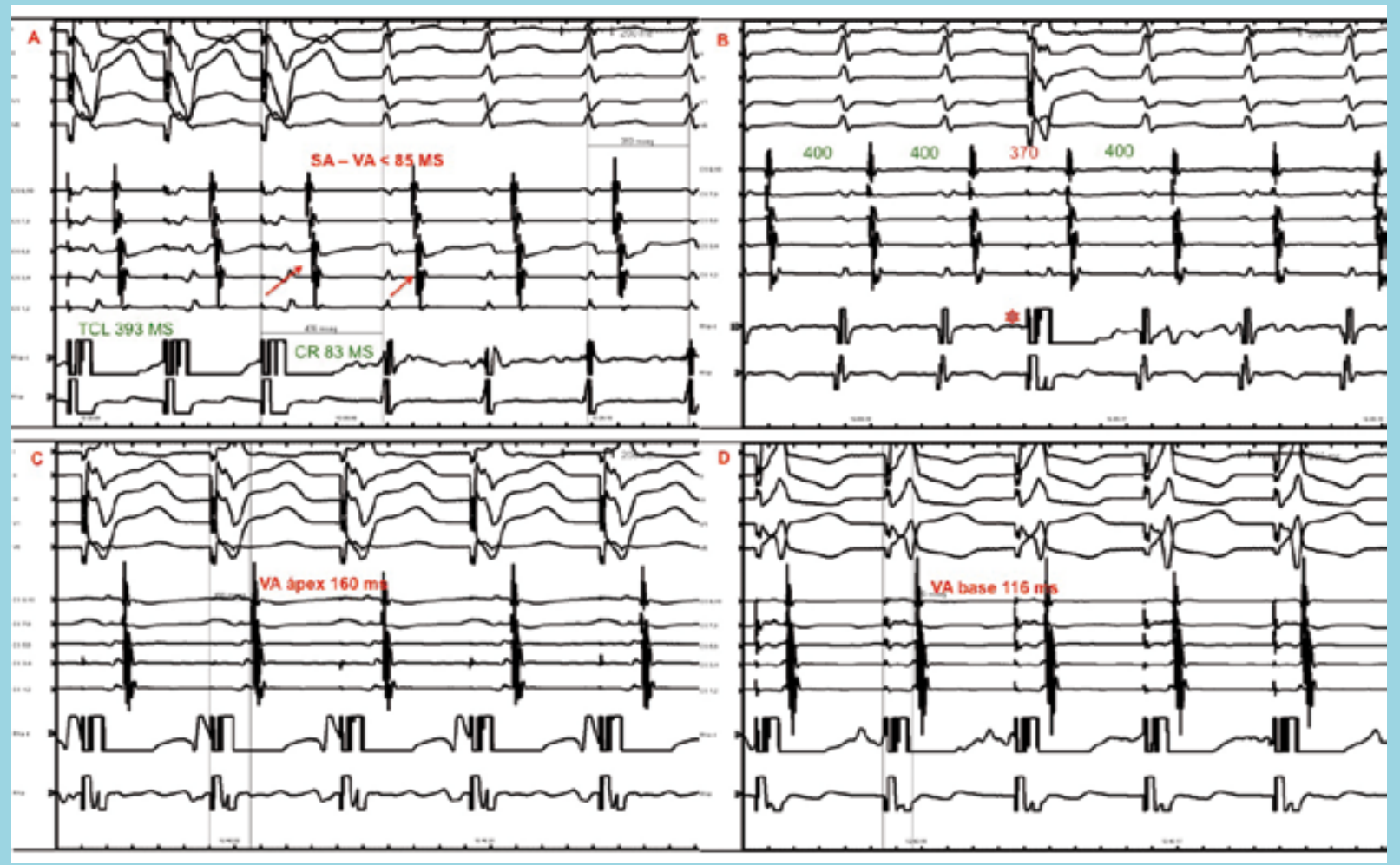

Maniobras electrofisiológicas para el diagnóstico diferencial de TPSV. A y B durante taquicardia, C y D en ritmo sinusal.

A. Encarrilamiento de la taquicardia desde ápex de VD mostró una respuesta $V-A-V$, un $S A-V A$ menor a 85 ms y un ciclo de retorno - ciclo de la taquicardia = 83 ms. Introducción de un extraestímulo ventricular durante His refractario (asterisco rojo), adelanta la aurícula siguiente sin interrumpir la taquicardia

C. Estimulación en ápex de VD muestra un intervalo VA $160 \mathrm{~ms}$ (cáliper)

D. Estimulación en Base de VD muestra un intervalo VA 116 ms (cáliper)

\section{Figura 4}

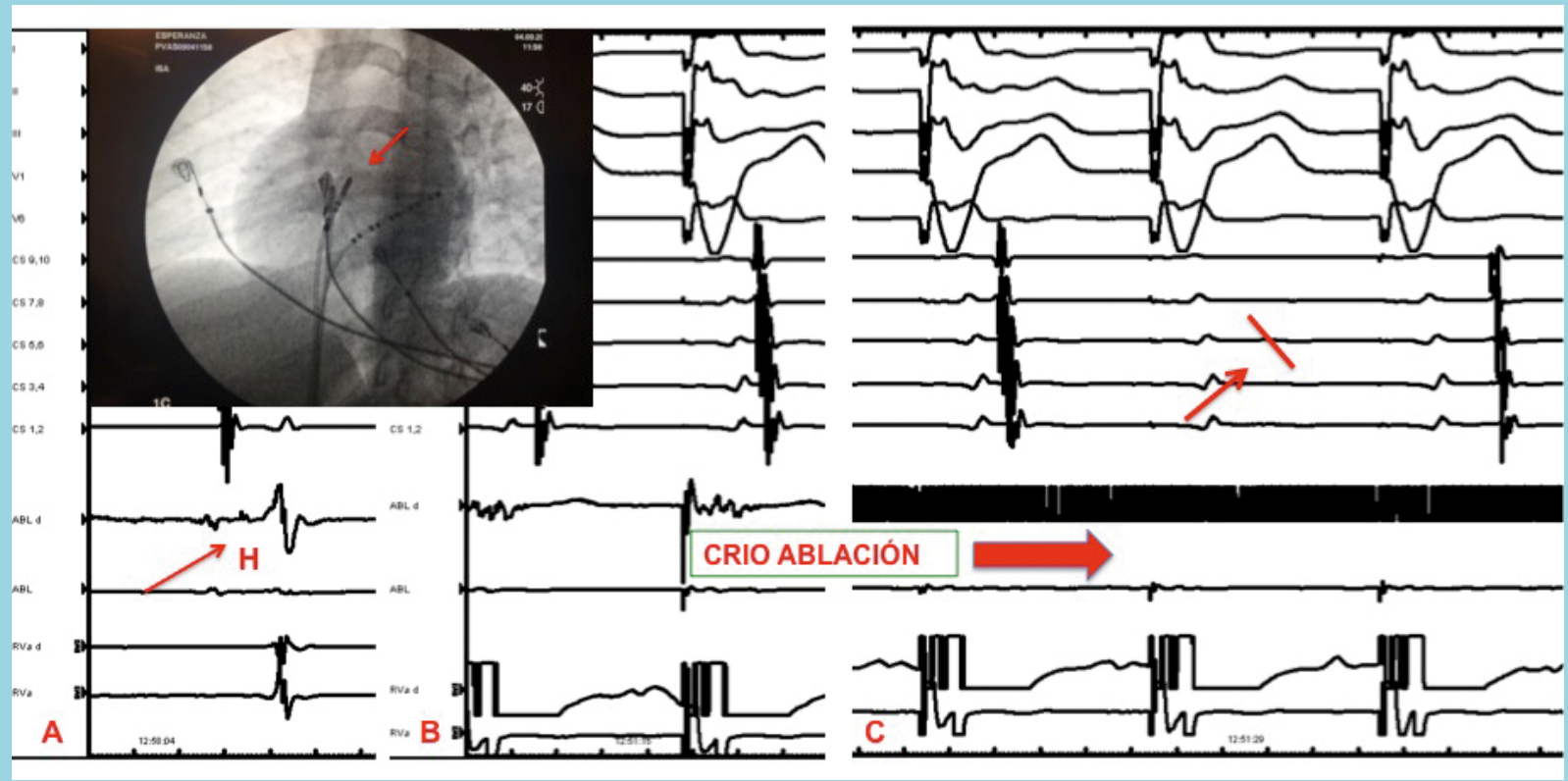

Registro intracavitario de la zona paraHisiana de crioablación

A. Registro intracavitario en ritmo sinusal de la zona de éxito. Flecha roja en registro indica señal de His (H) y flecha roja en radioscopía indica posición del catéter de crioablación. B. Registro intracavitario durante estimulación ventricular mostrando fusión VA y actividad fragmentada en canal ABLd

C. Registro intracavitario durante estimulación ventricular en crioablación. Flecha y línea roja indica momento de la interrupción en la conducción VA por la vía accesoria oculta. 
tal y anterior durante taquicardia y estimulación ventricular derecha mostró una localización parahisiana.

Dada la edad de la paciente y los riesgos de bloqueo aurículo ventricular que conlleva la ablación con radiofrecuencia en esta localización, se optó por realizar una crioablación (crio) de la vía accesoria parahisiana. Se avanzó un catéter específico para crioablación Frezzor Xtra $6 \mathrm{~mm}$ (Medtronic inc $(\mathcal{C})$ a la región parahisiana logrando observar, durante taquicardia, una actividad eléctrica fraccionada y continua en posición con registro de His. Se conectó a consola y se aplicó crioablación directa durante estimulación ventricular intermitente (para monitorear la indemnidad de la conducción anterógrada a través del nodo AV) logrando disociación VA a los 8 segundos de la aplicación y alcanzando temperatura de hasta $-75^{\circ} \mathrm{C}$ por 240 segundos. Tras detener la crioablación y al cabo de algunos minutos se observó reconducción VA a través de la vía accesoria. Se realizó una segunda crioablación por 240 segundos con igual resultado y recurrencia temprana tras el periodo de observación. Luego de una tercera aplicación de 360 segundos se logró evitar la reconducción por 45 minutos de observación (Figura 4A, B y C). El procedimiento no presentó complicaciones y la paciente se encuentra libre de episodios de TPSV al cabo de 12 meses de seguimiento.

\section{Discusión:}

El uso del frío como herramienta terapéutica se remonta al tiempo de Hipócrates (460 - 377 A.C) como mecanismo de alivio del dolor y antiinflamatorio. En 1899 el Dr. Campbell White utilizó refrigerantes para tratar variadas condiciones como lupus eritematoso, herpes zoster, carcinomas y epiteliomas. De Quervain trató con éxito en 1917 papilomas y cáncer de la vejiga. En 1913 el Dr. Iving Cooper desarrolló un "probe" de nitrógeno líquido capaz de alcanzar temperatura de $-196^{\circ} \mathrm{C}$ y lo utilizó en el tratamiento de la enfermedad de Parkinson y en el cáncer irresecable. En la actualidad, la crioterapia es una técnica desarrollada y aceptada para el tratamiento o coadyuvancia de tumores sólidos hepáticos, prostáticos, renales, etc ${ }^{1}$.

En cardiología, la crioablación de arritmias cardíacas se utiliza como alternativa a la radiofrecuencia para el tratamiento intervencionista de la fibrilación auricular y como una forma segura de tratar algunas arritmias cuyo sustrato se localice en la región del nodo AV (taquicardia intranodal), en arritmias cercana al nodo aurículo ventricular o haz de His. También puede ser de utilidad en arritmias auriculares focales con posicionamiento inestable, aprovechando la adhesión que se genera durante la aplicación de frío.

La tecnología fue desarrollada en el Montreal Heart Institute a finales de los noventa y fue adoptada con éxito en Europa en el 2001 y en USA en el 2005 luego de la publicación del Frosty Trial ${ }^{2}$, estudio que evaluó la eficacia y seguridad de la crio ablación mediante el catéter focal Frezzor. El estudio fue realizado en 166 pacientes y el objetivo de la ablación fue la vía lenta del nodo AV en 103 pacientes con reentrada del nodo AV (grupo 1), una vía accesoria en 51 pacientes con taquicardias ortodrómicas (grupo 2) y la unión AV en 12 pacientes con fibrilación auricular (grupo 3). El éxito agudo promedio fue de $83 \%$ y según sustrato, $91 \%$, $69 \%$ y $67 \%$ respectivamente $(\mathrm{p}<.001)$. En los pacientes con buen resultado inicial, el éxito a 6 meses fue de $91 \%$. Ninguno de los pacientes de los grupos 1 y 2 requirieron marcapasos.

En el estudio de Pérez -Castellanos y $\mathrm{col}^{3}$, en una población de pacientes referidos para ablación del nodo aurículo ventricular, se realizó crioablación en diferentes segmentos de la región medio y anteroseptal sin conseguir bloqueo persistente de la conducción anterógrada a través del NAV y haz de His, confirmando de esta forma, la seguridad de la técnica en sustratos perinodales y parahisianos.

Rodríguez y col randomizaron 119 pacientes con taquicardia por reentrada en el nodo AV a ablación con $\mathrm{RF}$ versus Crioablación ${ }^{4}$. No observaron diferencias en el éxito agudo del procedimiento; sin embargo, la tasa de recurrencia fue mayor en el grupo de crioablación ( $15 \%$ versus $3,4 \%, \mathrm{p}=0.03$ ). Un paciente del grupo $\mathrm{RF}$ requirió marcapasos definitivo.

El estudio ICY - AVNRT, recientemente publicado, incluyó 397 pacientes con taquicardia por reentrada del nodo AV. Se realizó crioablación de la vía nodal lenta con catéter Frezzor de $6 \mathrm{~mm}$, consiguiendo un éxito agudo de $95 \%$ y $93 \%$ a 6 meses 5 .

En el estudio de Bastani y col se trataron 27 pacientes consecutivos con vías accesorias AV anteroseptales (18) y septales medias (9). Se utilizó catéter Frezzor $6 \mathrm{~mm}$ y criomap a $-30^{\circ}$ previo a crioablación a $-80^{\circ}$ por 240 segundos. El éxito agudo fue $96 \%$, el tiempo de procedimiento y de radioscopía fue $163+/-61$ y $30+/-22$ min respectivamente. Durante el seguimiento promedio de $996+/-511$ días la recurrencia fue $27 \%$. Cinco de los 7 pacientes que recurrieron fueron a un segundo procedimiento de crioablación exitoso, dando 
un total de éxito de $89 \%$. La tasa de recurrencia fue mayor en aquellos pacientes que tuvieron un bloqueo mecánico de la vía accesoria durante el procedimiento $(86 \% \text { vs } 25 \%, \mathrm{p}=0.006)^{6}$.

Alonso -García describe los resultados de una serie de 224 pacientes pediátricos referidos para ablación con una mediana de 12,2 años de edad. Los sustratos más frecuentemente tratados fueron las vías accesorias (70\%; más del 50\% septales). El 35\% de los casos se realizaron con crioablación. El éxito agudo no presentó diferencia entre radiofrecuencia y crioablación (94,2\% vs 93,4); sin embargo, el éxito total a largo plazo fue significativamente mayor con radiofrecuencia $(84,8 \%$ vs $65,9 \%)^{7}$.

Un meta análisis comparó la eficacia y seguridad de la crioablación y de radiofrecuencia en el tratamiento de vías accesorias septales. De 4244 vías septales, 3495 fueron tratadas con radiofrecuencia y 749 con crioablación. El éxito agudo y a largo plazo fue de $89 \%$ y $88,4 \%$ respectivamente para RF y $86 \%$ y $75,9 \%$, respectivamente, para crio. La tasa de recurrencia fue $9,9 \%$ para RF y $18,1 \%$ para crio. El bloqueo AV persistente fue $2,7 \%$ en RF y no lo hubo en crioablación ${ }^{8}$. La información disponible sugiere que, si bien la crioablación en sustratos nodales o perihisianos tiene mayor tasa de recurrencia, puede ser considerada como una terapia de primera línea, especialmente en pacientes jóvenes, donde el riesgo de bloqueo AV y necesidad de estimulación permanente es relevante. Esta es la razón por la que utilizamos esta técnica en nuestra paciente. Utilizamos crioablación directa y no criomap debido a que ambas estrategias están validadas en la literatura cuando se dispone de una buena metodología para evaluar la conducción anterógrada por el nodo AV (en nuestro caso realizamos interrupciones de la estimulación ventricular cada 5 seg para evaluar los parámetros de conducción AV: AH y $\mathrm{HV}$ en ritmo sinusal). Uno de los factores a considerar durante la ablación es el tiempo al efecto de bloquear la conducción por la vía accesoria. En un análisis multivariado se observó que el único predictor independiente para la tasa de recurrencia fue el tiempo al efecto $<10$ segundos $(\mathrm{P}<.001)$. Una mayor tasa de recurrencia fue asociada con vías accesorias ocultas y a ablación durante taquicardia supraventricular $(\mathrm{P}<.03$ y $\mathrm{P}<.05$ respectivamente $)$. La experiencia del operador es otro factor que influye. En esta serie de pacientes pediátricos la recurrencia disminuyó desde $40 \%$ en los primeros 10 casos a $8,1 \%$ en los siguientes $37(\mathrm{P}<.02)^{9}$.

En la actualidad, el principal uso de la crioablación está en el área del tratamiento de la fibrilación auricular paroxística, mediante el uso de un balón que se infla con óxido nitroso a nivel ostial o antral de cada vena pulmonar generando una lesión transmural por congelamiento entre -40 y $-55^{\circ} \mathrm{C}$, con resultado de desconexión eléctrica bidireccional de las venas pulmonares. El estudio Fire and Ice comparó la eficacia y seguridad, en pacientes con fibrilación auricular paroxística, del aislamiento de venas pulmonares mediante la ablación punto a punto con radiofrecuencia y la crioablación con balón, concluyendo que no existían diferencias significativas entre ambas técnicas ${ }^{10}$. 


\section{Referencias}

1. COOPER (2001). "The History of Cryosurgery". J R Soc Med. 94 (4): 196-201

2. FRIEDMAN PL, DUBUC M, GREEN MS, JACKMAN WM, KEANE DT. Catheter cryoablation of supraventricular tachycardia: results of the multicenter prospective "frosty" trial. Heart Rhythm. 2004 Jul;1(2):129-38

3. PEREZ-CASTELLANO N, VILLACASTIN J, MORENO J, ISA R, RUIZ E, DOBLADO M, et al. High resistance of atrioventricular node to cryoablation: a great safety margin targeting perinodal arrhythmic substrates. Heart Rhythm 2006 oct; 3(10): $1189-95$

4. RODRIGUEZ - ENTEM FJ, EXPÓSITO V, GONZALEZ ENRIQUEZ S, OLALLA-ANTOLIN JJ. Cryoablation versus radiofrequency ablation for the treatment of atrioventricular nodal reentrant tachycardia: results of a prospective randomized study. J Interv Card Electrophysiol. 2013 Jan;36(1):41-5

5. WELLS P, DUBUC M, KLEIN GJ, DAN D, ROUX JF et al. Intracardiac ablation for atrioventricular nodal reentry tachycardia using a $6 \mathrm{~mm}$ distal electrode cryoablation catheter: Prospective, multicenter, North American study (ICY-AVNRT STUDY). J Cardiovasc Electrophysiol. 2018 Jan;29(1):167-176
6. BASTANI H, INSULANDER P, SCHWIELER J, TABRIZI F, BRAUNSCHWEIG F, KENNEBÄCK G, et al. Cryoablation of superoparaseptal and septal accessory pathways: a single centre experience. Europace. 2010 Jul;12(7):972-7.

7. ALONSO-GARCÍA A, ATIENZA F, ÁVILA P, UGUETO C, CENTENO M, ÁLVAREZ R, et al. Ablación pediátrica con catéter: características y resultados del procedimiento en un centro terciario de referencia. Rev esp Cardiol. 2018; 71: 794-800

8. BRAVO L, ATIENZA F, EIDELMAN G, ÁVILA P, PELLIZA M, CASTELLANO E, et al. Safety and efficacy of cryoablation vs. radiofrequency ablation of septal accessory pathways: systematic review of the literature and meta-analyses. Europace. 2018 Aug 1;20(8):1334-1342

9. SWISSA M, BIRK E, DAGAN T, FOGELMAN M, EINBINDER T, BRUCKHEIMER E, et al. Cryotherapy ablation of parahisian accessory pathways in children. Heart Rhythm. 2015 May;12(5):917-25

10. KUCK KH, FÜRNKRANZ A, CHUN KR, METZNER A, OUYANG F, SCHLÜTER M, et al. Cryoballoon or radiofrequency ablation for symptomatic paroxysmal atrial fibrillation: reintervention, rehospitalization, and quality-of-life outcomes in the FIRE AND ICE trial. Eur Heart J. 2016 Oct 7;37(38):2858-2865 